\title{
A cm-wave excess over free-free emission in planetary nebulae
}

\author{
Simon Casassus ${ }^{1} \dagger$, Lars- $\AA$ ke Nyman ${ }^{2}$, A. C. S. Readhead ${ }^{3}$ \\ \& Tim Pearson ${ }^{3}$ \\ ${ }^{1}$ Departamento de Astronomía, Universidad de Chile, Casilla 36-D, Santiago, Chile \\ email: simon@das.uchile.cl \\ ${ }^{2}$ European Southern Observatory, Casilla 19001, Santiago 19, Chile \\ ${ }^{3}$ Onsala Space Observatory, 43992 Onsala, Sweden \\ ${ }^{4}$ Owens Valley Radio Observatory, California Institute of Technology, Pasadena, CA 91125
}

\begin{abstract}
A byproduct of experiments designed to map the CMB is the detection of a new component of foreground galactic emission. The anomalous foreground at 10-30 GHz, unexplained by traditional emission mechanisms, correlates with $100 \mu \mathrm{m}$ dust emission, and is thus presumably due to dust. We present evidence obtained with the CBI and SIMBA+SEST supporting the existence of a $31 \mathrm{GHz}$ excess over free-free emission in PNe. Possible interpretations involve a spinning dust component or $1 \mathrm{~mm}$ extinction due to metallic needles.
\end{abstract}

Keywords. radiation mechanisms: general, planetary nebulae: general, radio continuum: ISM, submillimetre

An increasing amount of evidence supports the existence of a new continuum emission mechanism in the diffuse interstellar medium (ISM) at 10-30 GHz (Leitch et al. 1997). As proposed by Draine \& Lazarian (1998) a candidate emission mechanism is electric dipole radiation from spinning very small grains (VSGs), or spinning dust. Examples in specific objects have been found by Finkbeiner (2004), Casassus et al. (2004), Watson et al. (2005), Casassus et al. (2006). Here we report on CBI and SEST+SIMBA observations of PNe.

The CBI is an interferometer array with 13 antennas mounted on a $6 \mathrm{~m}$ tracking platform. Its synthesized beam is $\sim 6$ arcmin, and it covers $26-36 \mathrm{GHz}$ in $10 \times 1 \mathrm{GHz}$ channels. We extracted flux densities by fitting parametrized models, discarding PNe with contaminating emission within the primary beam of 45 arcmin FWHM, as revealed by reduced $\chi^{2}$ higher than $\sim 1.5$. Independent integrations on different nights corroborate the uncertainties. Spectral indices over $26-36 \mathrm{GHz}$ are all consistent with free-free emission, with $\alpha=-0.15\left(F(\nu)=F\left(\nu_{\circ}\right)\left(\nu / \nu_{\circ}\right)^{\alpha}\right)$. A comparison with $5 \mathrm{GHz}$, mostly taken from Zijlstra et al. (1989), follows thin free-free emission, within the $10 \%$ calibration uncertainty of the literature data. Exceptions are Hen2-142, M2-9, and SwSt 1, with 3, 5, and $8 \sigma$ excesses at $31 \mathrm{GHz}$. The average of $5-31 \mathrm{GHz}$ indices is $\left\langle\alpha_{5}^{31}\right\rangle=-0.03 \pm 0.06$.

The Sest IMaging Bolometer Array (SIMBA) operates at $1.2 \mathrm{~mm}\left(250 \mathrm{GHz}, 24^{\prime \prime}\right.$ resolution). We observed 35 PNe during 3 different observing runs in 2001 and 2002, and extracted flux densities by integrating the sky intensity in a 0.6 arcmin radius. The uncertainty incorporates in a quadratic sum a $10 \%$ calibration error. The SIMBA maps confirm previous pointed heterodyne measurements obtained by L.-Å. Nyman. Our flux densities are consistent with those at $1.1 \mathrm{~mm}$ from Hoare et al. (1990), within $2 \sigma$, for the four objects we have in common (NGC 6572, NGC 6302, M2-9, and NGC 6537).

$\dagger$ S.C. acknowledges support from FONDECYT 1060827 and FONDAP 15010003. 


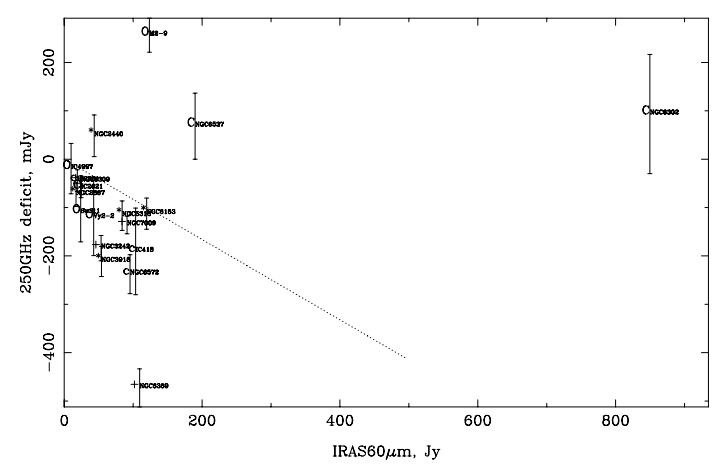

Figure 1. The $y$-axis shows the difference between the SIMBA flux density and the free-free level extrapolated from $31 \mathrm{GHz}$, and the $x$-axis shows IRAS $60 \mu \mathrm{m}$ flux density. NGC 6302 and M 2-9 are excluded for clarity.

All CBI/SIMBA objects show a deficit at $250 \mathrm{GHz}$ over the free-free level extrapolated from $31 \mathrm{GHz}$, with the exception of M2-9 ( $7 \sigma$ excess). The excess $31 \mathrm{GHz}$ emission represents $\sim 30-50 \%$. Fig. 1 plots the $250 \mathrm{GHz}$ deficit against IRAS $60 \mu \mathrm{m} \dagger$. The cm-wave excess does not seem related to the $10 \mu \mathrm{m}$ dust emission features. The $250 \mathrm{GHz}$ deficit is still significant when extrapolating the free-free level from $5 \mathrm{GHz}$, albeit for less objects, which may be due in part to the $10 \%$ calibration uncertainty of the $5 \mathrm{GHz}$ data, but also to a $5-31 \mathrm{GHz}$ spectral index $>-0.15$.

Is it spinning dust? If spinning dust emission accounts for $30 \%$ of the $31 \mathrm{GHz}$ flux density, then $\alpha_{5}^{31}=0.045$, which is consistent with $\left\langle\alpha_{5}^{31}\right\rangle$ within $2 \sigma$. Spinning dust drops above $30 \mathrm{GHz}$, and is $>300$ times weaker at $100 \mathrm{GHz}$.

A synchrotron component? On average the $31-250 \mathrm{GHz}$ spectral index is $\left\langle\alpha_{31}^{250}\right\rangle=$ $-0.36 \pm 0.06$, with an rms scatter of 0.16 . But free-free absorption with a turn-over frequency at $40 \mathrm{GHz}$ requires an absurd $T_{e}<600 \mathrm{~K}$ for an emission measure of $10^{6} \mathrm{~cm}^{-6} \mathrm{pc}$.

The $250 \mathrm{GHz}$ deficit could be interpreted as extinction from metallic needles. Using the formulae from Dwek (2004), a long-wavelength cutoff for the gray extinction of $\lambda_{\circ}=1 \mathrm{~mm}$ requires a needle aspect ratio $l / a$ of $\sim 8000$ for a resistivity of $\rho_{R}=10^{-6} \Omega \mathrm{cm}$. A unit needle opacity at $1 \mathrm{~mm}$ gives a total needle mass of $210^{-6} \mathrm{M}_{\odot}$ for a grain material density of $\rho_{m}=8.15 \mathrm{~g} \mathrm{~cm}^{-3}$.

\section{References}

Draine, B.T., \& Lazarian, A., 1998, ApJ, 508, 157

Casassus, S., et al., 2006, ApJ, 639, 951

Casassus,S., et al., 2004, ApJ, 603, 599

Casassus, S., Roche, P. F., Aitken, D. K., \& Smith, C. H., 2001, MNRAS, 320, 424

Dwek, E., 2004, ApJ, 607, 848

Finkbeiner, D. P., 2004, ApJ, 614, 186

Hoare, M. G., Roche, P. F., \& Clegg, R. E. S., MNRAS, 258, 257

Leitch, E.M., Readhead, A.C.S., Pearson, T.J., \& Myers, S.T., ApJL 486, L23

Watson, R. A., et al., 2005, ApJ, 624, 89

Zijlstra et al. 1989, A\&AS, 79, 329

$\dagger$ The $10 \mu \mathrm{m}$ dust emission features compiled from the literature are indicated by ' $\mathrm{C}$ ' for PAHs, 'c' for $\mathrm{SiC}$, 'O' for silicates, '+' for weak and featureless continuum, and '*' when no data are available (data from Casassus et al. (2001)) 